\title{
Quantitative Comparison of Organic Photovoltaic Bulk Heterojunction Photostability Under Laser Illumination
}

\author{
Michael D. Lesoine, ${ }^{\dagger, \ddagger, \#}$ Jonathan M. Bobbitt, ${ }^{\dagger, \ddagger \#}$ John A. Carr, ${ }^{\S}$ Moneim Elshobaki, ${ }^{\|, \perp}$ \\ Sumit Chaudhary, ${ }^{\S, \| l}$ and Emily A. Smith $*,,,+$ \\ ${ }^{\dagger}$ U.S. Department of Energy, Ames Laboratory, Ames, Iowa 50011, United States \\ ${ }^{\ddagger}$ Department of Chemistry, Iowa State University, 1605 Gilman Hall, Ames, Iowa 50011, United States \\ ${ }^{\S}$ Department of Electrical and Computer Engineering, Iowa State University, 2215 Coover Hall, Ames, Iowa 50011, United States \\ "Department of Materials Science and Engineering, Iowa State University, 2220 Hoover Hall, Ames, Iowa 50011, United States \\ ${ }^{\perp}$ Department of Physics, Mansoura University, Mansoura, 35516, Egypt
}

Supporting Information

ABSTRACT: The photostability of bulk heterojunction organic photovoltaic films containing a polymer donor and a fullerene-derivative acceptor was examined using resonance Raman spectroscopy and controlled laser power densities. The polymer donors were poly(3-hexylthiophene-2,5-diyl) (P3HT), poly[[9-(1-octylnonyl)-9Hcarbazole-2,7-diyl]-2,5-thiophenediyl-2,1,3-benzothiadiazole-4,7-diyl-2,5-thiophenediyl ] (PCDTBT), or poly $\left(\left\{4,8\right.\right.$-bis [(2-ethylhexyl)oxy]benzo[1,2-b:4,5- $\left.\mathrm{b}^{\prime}\right]$ dithiophene-2,6diyl $\}\{3$-fluoro-2-[(2-ethylhexyl)carbonyl $]$ thieno $[3,4-b]$ thiophenediyl $\})$ (PTB7). Four

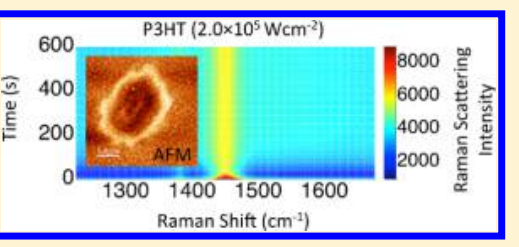
sample preparation methods were studied: (i) thin or (ii) thick films with fast solvent evaporation under nitrogen, (iii) thick films with slow solvent evaporation under nitrogen, and (iv) thin films dried under nitrogen followed by thermal annealing. Polymer order was assessed by monitoring a Raman peak's full width at half-maximum and location as a function of illumination time and laser power densities from $2.5 \times 10^{3}$ to $2.5 \times 10^{5} \mathrm{~W} \mathrm{~cm}^{-2}$. Resonance Raman spectroscopy measurements show that before prolonged illumination, PCDTBT and PTB7 have the same initial order for all preparation conditions, while P3HT order improves with slow solvent drying or thermal annealing. All films exhibited changes to bulk heterojunction structure with $2.5 \times$ $10^{5} \mathrm{Wcm}^{-2}$ laser illumination as measured by resonance Raman spectroscopy, and atomic force microscopy images show evidence of sample heating that affects the polymer over an area greater than the illumination profile. Photostability data are important for proper characterization by techniques involving illumination and the development of devices suitable for real-world applications.

\section{INTRODUCTION}

Organic photovoltaic devices (OPVs) fabricated from polymer donors and fullerene-derivative acceptors are a promising portable and renewable source of electricity. ${ }^{1,2}$ A common OPV design has a transparent substrate, a transparent anode, anodic buffer layer, an active layer containing a donor mixed with acceptor, a cathodic buffer layer and finally a cathode. ${ }^{2}$ Other designs have been reported. ${ }^{2,3}$ The morphology and order of the active layer affect OPV performance. ${ }^{4,5}$ A bound electronhole pair (exciton) is created upon absorption of light by the donor. Bulk heterojunction morphology consists of a highly ordered polymer donor with a interpenetrating bicontinuous acceptor structure that helps facilitate the dissociation of an exciton at the interface between the donor and acceptor. Donor/acceptor domains of the size of the exciton diffusion length, typically less than $10 \mathrm{~nm}$, are essential to prevent the majority of the excitons from decaying. ${ }^{6}$ Morphology affects, among other properties, electronic defects (e.g., distortions in the geometry of $\mathrm{sp}^{2}$ carbon covalent bonds in the $\pi$-conjugated polymers' backbone), charge mobility (i.e., electron transport efficiency), and absorption (e.g., shift to longer wavelengths with increasing polymer order). ${ }^{7-9}$
Performance metrics for OPV devices typically focus on how efficiently a constructed device converts solar radiation into electricity. Of equal importance is the development of stable OPVs with long operating lifetimes. ${ }^{10-18}$ One reported case showed that OPV stability increases when low molecular weight organic contaminants are removed from the polymer PBDTTPD (poly[[5-(2-ethylhexyl)-5,6-dihydro-4,6-dioxo-4Hthieno[3,4-c] pyrrole-1,3-diyl][4,8-bis[(2-ethylhexyl)oxy]benzo[1,2-b:4,5-b'] dithiophene-2,6-diyl]]). ${ }^{14}$ The short-circuit current density of cells stored in dark and inert conditions drops only $6 \%$ after 111 days when size exclusion chromatography is used to reduce low molecular weight species. Fullerene-based acceptors containing bulky substituents to suppress crystallinity increased thermal stability. Devices fabricated from these acceptors can be maintained at $150{ }^{\circ} \mathrm{C}$ up to $10 \mathrm{~h}$ without degradation of device efficiency. ${ }^{10}$ In addition to altering the composition of the donor and acceptor, OPVs are being optimized with different preparation conditions. For example

Received: September 22, 2014

Revised: November 20, 2014

Published: November 20, 2014 
thermal $^{10,13,19,20}$ and solvent ${ }^{21}$ annealing can improve device performance for poly(3-hexylthiophene-2,5-diyl) (P3HT)based OPVs. Treatment of the hole transport layer with ultraviolet light is reported to improve OPV stability as a result of increased wettability and contact with the active layer. ${ }^{15}$ These selected examples may not extend to other systems; degradation mechanisms are known to be complex and largely material specific.

Raman spectroscopy can be used to measure chemical and structural properties of the OPV active layer. ${ }^{21-25}$ Reported indicators of order in thiophene-based bulk heterojunction OPVs are the location and full width at half-maximum (fwhm) of the thiophene symmetric stretch. ${ }^{21,24-27}$ Smaller fwhm or Raman shifts are reported to correspond to an increase in the device efficiency. ${ }^{21,23-25}$ Furukawa reported the Raman spectra of powdered and solution $\mathrm{P} 3 \mathrm{HT}$ with maximum intensities at 1450 and $1470 \mathrm{~cm}^{-1}$, respectively. ${ }^{28,29}$ The solution should exhibit relative disorder while the powder is expected to exhibit relative order. A recent publication by Wood et al. showed negligible shifts in the thiophene peak maximum with heating up to at least $140{ }^{\circ} \mathrm{C}$ and a shift of approximately $15 \mathrm{~cm}^{-1}$ only after annealing the film at $\sim 200{ }^{\circ} \mathrm{C}$. ${ }^{30}$

We report photostability measurements of bulk heterojunction thin films prepared with one of three polymer donors and one of four sample preparation methods using resonance Raman spectroscopy combined with controlled laser power densities. Raman spectroscopy is ideally suited for these measurements since the changes in the spectra over time simultaneously provide information about the polymer stability and the mechanism of degradation. For example, changes in the Raman peaks can be assigned to specific functional groups and spectral background can indicate changes in luminescence. Many previous Raman studies of OPV films do not report laser power densities or, when reported, used high laser power densities. This may affect the measurement of polymer order through sample heating, degradation, or a similar mechanism. Our work provides new information on the photostability of OPV thin films prepared with different active layer compositions and processing methods, while simultaneously elucidating the effect of laser power density on the measurement of polymer order.

\section{EXPERIMENTAL METHODS}

Film Preparation. Samples were generated with the strata shown in Figure 1.

All samples were prepared and annealed in a nitrogen environment. First glass substrates coated with a 120 to $160 \mathrm{~nm}$ layer of indium tin oxide (ITO) from Delta Technologies were spin coated with poly(3,4-ethylenedioxythiophene) and poly(styrenesulfonate) (PEDOT:PSS) (Clevios P VP AI 4083, Heraeus Precious Metals GmbH \& Co. KG, Leverkusen, Germany) at $4000 \mathrm{rpm}$ for $60 \mathrm{~s}$ to form a 30 to $40 \mathrm{~nm}$ layer. Next, a film of P3HT, poly[[9-(1-octylnonyl)-9H-carbazole2,7-diyl]-2,5-thiophenediyl-2,1,3-benzothiadiazole-4,7-diyl-2,5thiophenediyl] (PCDTBT), or poly $(\{4,8$-bis[(2-ethylhexyl)oxy]benzo[1,2-b:4,5-b'] dithiophene-2,6-diyl $\}\{3$-fluoro-2-[(2ethylhexyl)carbonyl $]$ thieno[3,4-b] thiophenediyl $\}$ ) (PTB7) (1Material, Dorval, Quebec, Canada) mixed with the acceptor $\mathrm{PC}_{x} \mathrm{BM}(x=60$ for P3HT and $x=70$ for PCDTBT and PTB7) was formed. The specific film compositions and spin coating parameters are shown in Table 1.

Four preparation methods were used with each donor: a slow or fast spin coating speed (which affects the film thickness)

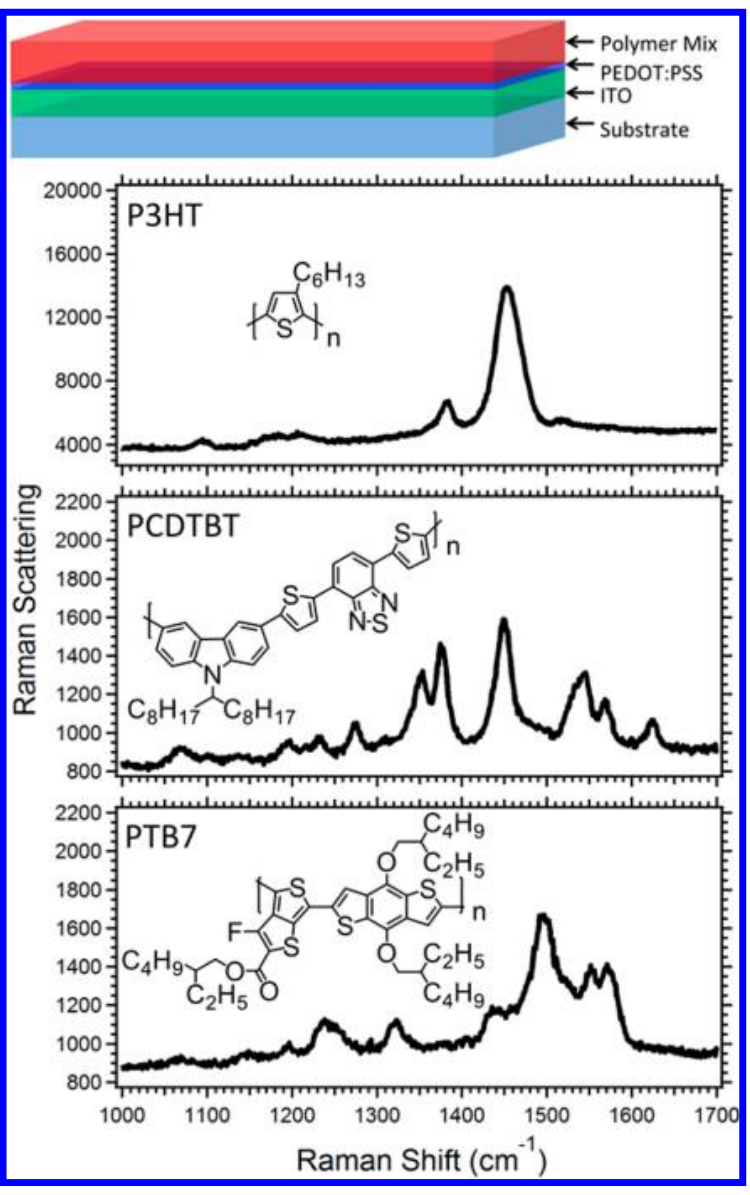

Figure 1. At the top is a schematic of the sample layout. Raman spectra of samples formed with the donor P3HT, PCDTBT, or PTB7 (as indicated by the label and structure) mixed with $\mathrm{PC}_{x} \mathrm{BM}$. P3HT $(180.6 \pm 0.7 \mathrm{~nm}$ thickness), PCDTBT $(180 \pm 10 \mathrm{~nm})$, and PTB7 $(160 \pm 10 \mathrm{~nm})$ films were prepared with solvent evaporation under nitrogen and measured with $2.5 \times 10^{3}(\mathrm{P} 3 \mathrm{HT})$ or $5.0 \times 10^{3} \mathrm{Wcm}^{-2}$ (PCDTBT and PTB7).

with drying under nitrogen, slow spin coating speed with slow solvent evaporation under nitrogen, and a fast spin coating speed with drying under nitrogen followed by thermal annealing for $10 \mathrm{~min}$. After initial sample preparation, the PCDTBT and PTB7 samples were stored under vacuum for 24 $\mathrm{h}$ before measurements were performed. The films were removed from the nitrogen environment, and all further analyses were performed at ambient laboratory conditions. The thickness of the films was measured after the Raman spectra were collected with a NewView 7100 Profilometer (Zygo, Middlefield, CT).

Spectral Measurements. A lab-built Raman microscope based on a DM IRBE platform (Leica, Wetzlar, Germany) with $532 \mathrm{~nm}$ laser excitation with a fwhm of $15.8 \pm 0.2 \mathrm{~cm}^{-1}$ (Sapphire SF $532 \mathrm{~nm} 150 \mathrm{~mW}$, Coherent, Santa Clara, CA) was used to perform the Raman measurements. The laser profile was expanded using a 10× beam expander (59-127, Edmund Optics, Barrington, NJ) in order to backfill a $10 \times$ magnification, 0.25 numerical aperture microscope objective (Leica) to achieve a laser spot with a diameter of $1.6 \pm 0.2 \mu \mathrm{m}$. Laser excitation powers at the sample were $50-5000 \mu \mathrm{W}$ corresponding to $2.5 \times 10^{3}$ to $2.5 \times 10^{5} \mathrm{~W} \mathrm{~cm}^{-2}$. The films were illuminated with $1.8 \times 10^{4}$ to $1.8 \times 10^{6}$ suns equivalent of $532 \mathrm{~nm}$ laser. The suns equivalent was determined by dividing 
Table 1. Sample Preparation Conditions and Measured Thickness for the Samples Included in This Study

\begin{tabular}{|c|c|c|c|c|c|c|c|c|}
\hline polymer & sample & ratio $^{a}$ & $\mathrm{RPM}^{b}$ & time $(s)^{c}$ & drying & thermal annealing & thickness $(\mathrm{nm})^{d}$ & fwhm $\left(\mathrm{cm}^{-1}\right)^{e}$ \\
\hline \multirow[t]{4}{*}{ P3HT } & fast dry thick & $1: 1$ & 600 & 40 & nitrogen & NA & $277 \pm 3$ & $34.1 \pm 0.7$ \\
\hline & slow dry thick & $1: 1$ & 600 & 40 & solvent $^{f}$ & NA & $278 \pm 5$ & $31.3 \pm 0.4$ \\
\hline & fast dry thin & $1: 1$ & 1000 & 40 & nitrogen & NA & $180.6 \pm 0.7$ & $34.73 \pm 0.07$ \\
\hline & fast dry thermal anneal thin & $1: 1$ & 1000 & 40 & nitrogen & $100{ }^{\circ} \mathrm{C} ; 600 \mathrm{~s}$ & $170 \pm 20$ & $32.2 \pm 0.3$ \\
\hline \multirow[t]{4}{*}{ PCDTBT } & fast dry thick & $1: 3.5$ & 600 & 40 & nitrogen & NA & $180 \pm 10$ & $22.2 \pm 0.6$ \\
\hline & slow dry thick & $1: 3.5$ & 600 & 40 & solvent ${ }^{f}$ & NA & $170 \pm 20$ & $22.2 \pm 0.3$ \\
\hline & fast dry thin & $1: 3.5$ & 1500 & 40 & nitrogen & NA & $111 \pm 8$ & $22.0 \pm 0.6$ \\
\hline & fast dry thermal anneal thin & $1: 3.5$ & 1500 & 40 & nitrogen & $80{ }^{\circ} \mathrm{C} ; 600 \mathrm{~s}$ & $81 \pm 3$ & $22.5 \pm 0.8$ \\
\hline \multirow[t]{4}{*}{ РTB7 } & fast dry thick & $1: 1.5$ & 600 & 60 & nitrogen & NA & $160 \pm 10$ & $20.9 \pm 0.8$ \\
\hline & slow dry thick & $1: 1.5$ & 600 & 60 & solvent $^{f}$ & NA & $190 \pm 10$ & $21 \pm 1$ \\
\hline & fast dry thin & $1: 1.5$ & 1000 & 60 & nitrogen & NA & $114 \pm 4$ & $22.1 \pm 0.8$ \\
\hline & fast dry thermal anneal thin & $1: 1.5$ & 1000 & 60 & nitrogen & $80{ }^{\circ} \mathrm{C} ; 600 \mathrm{~s}$ & $103 \pm 6$ & $21 \pm 1$ \\
\hline
\end{tabular}

${ }^{a}$ Ratio of polymer donor to $\mathrm{PC}_{x} \mathrm{BM}$ acceptor. ${ }^{b}$ Spin-coating speed, rotations per minute. ${ }^{c}$ Spin-coating duration. ${ }^{d}$ As determined by profilometry. ${ }^{e} 2.5 \times 10^{3} \mathrm{~W} \mathrm{~cm}^{-2} .{ }^{f}$ Solvent was 1,2 -dichlorobenzene, and $2 \mathrm{~h}$ elapsed during drying under nitrogen.

the laser power density (at $532 \mathrm{~nm}$ ) by the integrated power density of the sun over all wavelengths. The objective was used to both focus the laser for excitation as well as collect the Raman scattering signal from the epi-direction. The Raman signal was focused with a lens onto a HoloSpec f/1.8i spectrograph (Kaiser Optical Systems, Ann Arbor, MI) and directed to a charged coupled device (CCD) (Newton 940, Andor Technology, Belfast, UK) with $2048 \times 512$ pixels.

Raman spectra were obtained at ambient laboratory conditions using Solis software version 4.23.30002.0 (Andor Technology). Collection times between 1 to $150 \mathrm{~s}$ were used depending on the excitation power in order to maintain a signal-to-noise ratio in the range of 40 to 75 throughout the measurements. Two collections were performed to facilitate cosmic ray removal. Raman images of P3HT samples were generated with a lab-developed program in Labview (2010 version, National Instruments, Austin, TX) to translate a sample on a XY-stage (ProScan, Prior Scientific, Rockland, $\mathrm{MA})$. A power density of $2.5 \times 10^{3} \mathrm{Wcm}^{-2}$ was used with a collection time of $15 \mathrm{~s}$ and a stage movement of $1 \mu \mathrm{m}$ per step.

Absorption spectra were collected using an Agilent 8453 UV-visible spectrophotometer (Santa Clara, CA). A sample without the active layer was used to collect a blank spectrum.

Data analysis. Raman spectra were analyzed using Igor Pro 6.3.2.3 (Wavemetrics, Lake Oswego, OR) batch-fit function with a Gaussian fit from 1325 to $1525 \mathrm{~cm}^{-1}$ for P3HT and PCDTBT and 1450 to $1550 \mathrm{~cm}^{-1}$ for PTB7. A linear baseline was used in all of the fits. The properties of a spectral peak, including full-width at half-maximum (fwhm) and location, were plotted as a function of time for the stability measurements. Luminescence, which appears as background in Raman spectra, was measured in a spectral region without Raman peaks $\left(2000 \mathrm{~cm}^{-1}\right)$.

\section{RESULTS AND DISCUSSION}

Bulk Heterojunction Thin Film Photostability Measured with Controlled Laser Power Densities and Resonance Raman Microscopy. The Raman spectra of films containing P3HT: $\mathrm{PC}_{60} \mathrm{BM}, \mathrm{PCDTBT}: \mathrm{PC}_{70} \mathrm{BM}$, or PTB7: $\mathrm{PC}_{70} \mathrm{BM}$ with thicknesses between 160 to $180 \mathrm{~nm}$ are shown in Figure 1. (Hereafter, only the polymer donor is used to identify the sample under study, and the acceptor is omitted for simplicity.) All three polymer donors have thiophene-based groups in their backbone, and exhibit resonantly enhanced

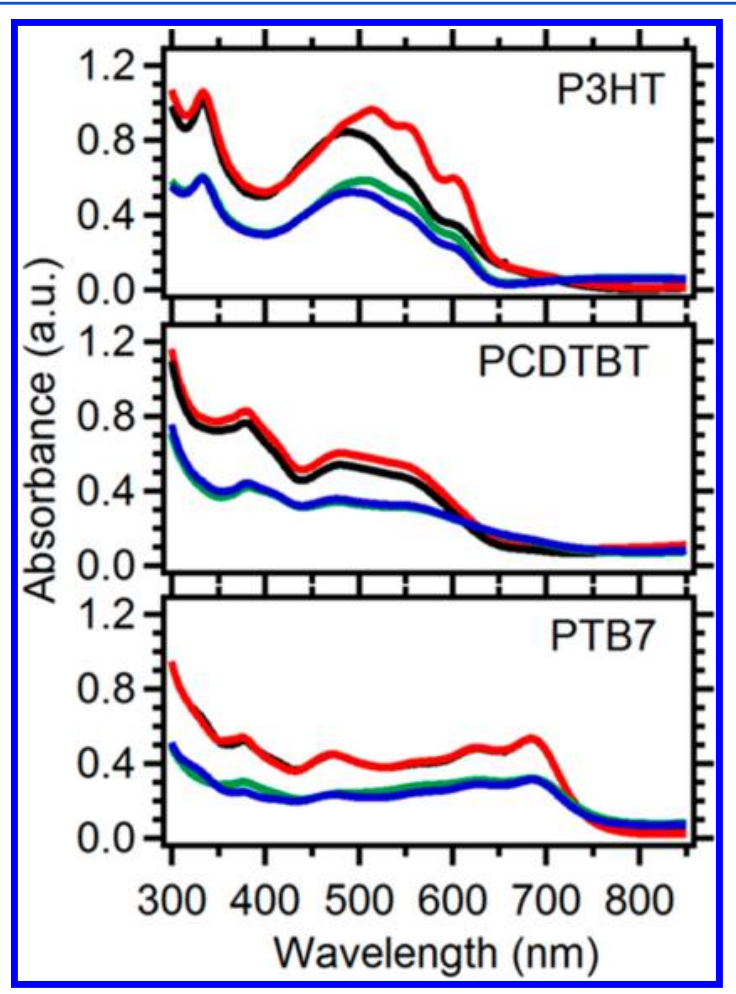

Figure 2. Absorption spectra for P3HT, РCDTBT, or PTB7 samples with the following preparation: thick film with solvent evaporation under nitrogen (black); thick film with slow solvent evaporation under nitrogen (red); thin film with solvent evaporation under nitrogen (blue); and a thin film dried under nitrogen followed by thermal annealing (green). Other sample preparation and thicknesses are shown in Table 1

Raman spectra using $532 \mathrm{~nm}$ excitation due to the appreciable absorption at this wavelength (Figure 2).

The most intense peak in each spectrum was used for subsequent stability measurements. These are the thiophene group at $\sim 1450 \mathrm{~cm}^{-1}$ for $\mathrm{P} 3 \mathrm{HT},{ }^{20,31}$ a broad ring stretch spanning the two thiophenes and the benzothiadiazole at $\sim 1447 \mathrm{~cm}^{-1}$ for PCDTBT, ${ }^{32}$ and the 3-fluorothiophene group at $\sim 1490 \mathrm{~cm}^{-1}$ for PTB7. ${ }^{33}$ The Raman spectrum of $\mathrm{PC}_{60} \mathrm{BM}$ and $\mathrm{PC}_{70} \mathrm{BM}$ has a peak at $1465 \mathrm{~cm}^{-1}$ that is not apparent in any of the spectra shown in Figure 1. This is expected since $\mathrm{PC}_{60} \mathrm{BM}$ and $\mathrm{PC}_{70} \mathrm{BM}$ have minimal absorption at $532 \mathrm{~nm}$ relative to the polymers, and also have a smaller Raman cross 


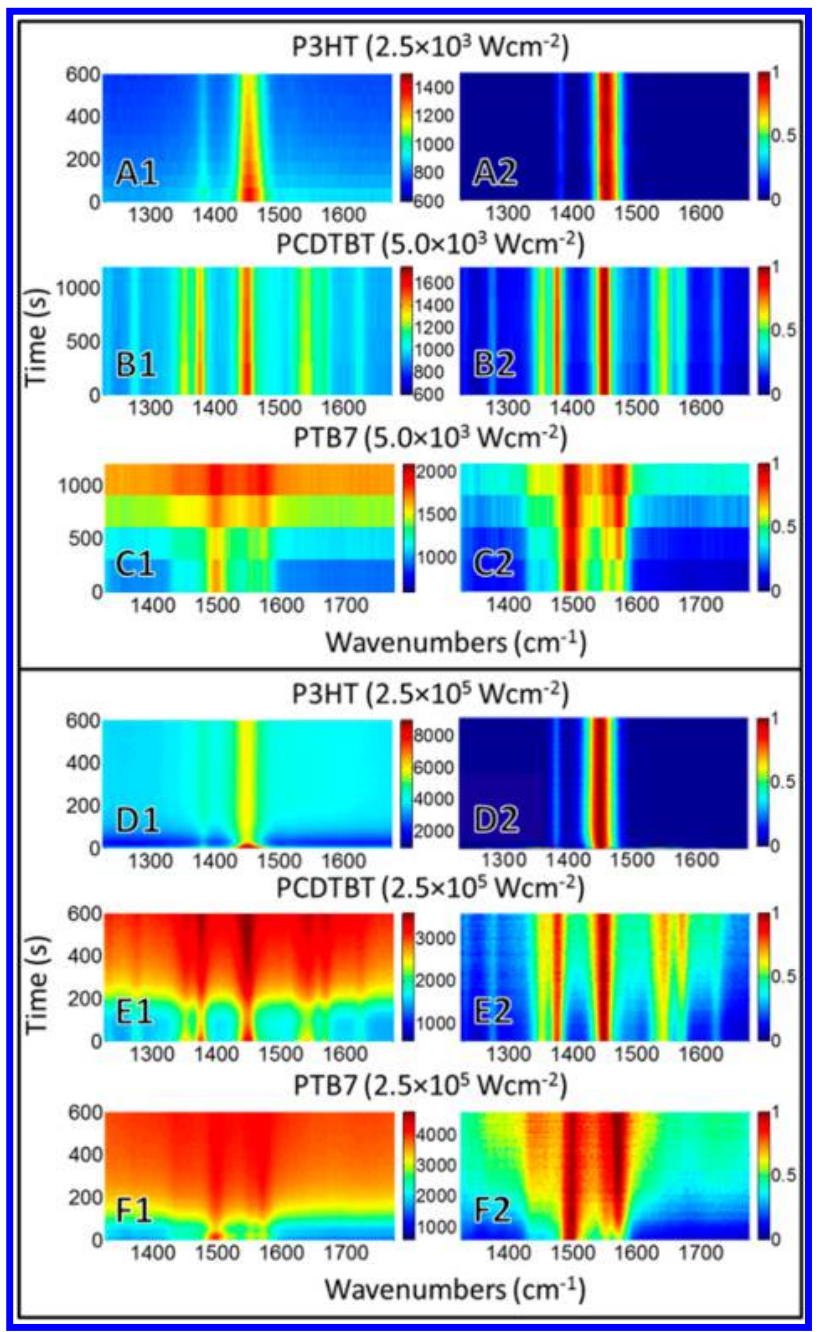

Figure 3. Raw (A1-F1) and normalized background subtracted (A2F2) Raman spectra versus illumination time for samples prepared with the indicated donor. The laser power density is listed at the top of each set of graphs. All samples were thick films with solvent evaporation under nitrogen (Table 1). The raw spectra show changes in peak intensity, including the generation of background luminescence, while the normalized spectra show changes in peak width and location. The color scale is Raman scattering intensity.

section compared to the polymers. ${ }^{26}$ Using the same collection parameters, $\sim 10 \times$ more signal is measured for P3HT compared to PCDTBT and PTB7. The latter two have similar signal intensities. In order to compare spectra with similar signal-tonoise ratios, the acquisition time for all subsequent Raman measurements was increased for PCDTBT and PTB7. This results in a decreased time resolution for photostability studies of these polymers compared to $\mathrm{P} 3 \mathrm{HT}$.

The samples prepared with fast solvent evaporation under nitrogen conditions were illuminated with power densities of $2.5 \times 10^{3}(\mathrm{P} 3 \mathrm{HT}), 5.0 \times 10^{3}$ (PCDTBT and PTB7), and $2.5 \times$ $10^{5} \mathrm{~W} \mathrm{~cm}^{-2}$ while simultaneously collecting Raman spectra to measure the photostability of the films over time (Figure 3).

The unprocessed spectra (Figure 3, left column) reveal changes in the peak and background intensity. The electronic transitions that occur when using a resonant laser wavelength can generate background luminescence in the Raman spectra. With the same experimental conditions as used to collect data shown in Figure 3, no Raman peaks are recorded for a solution

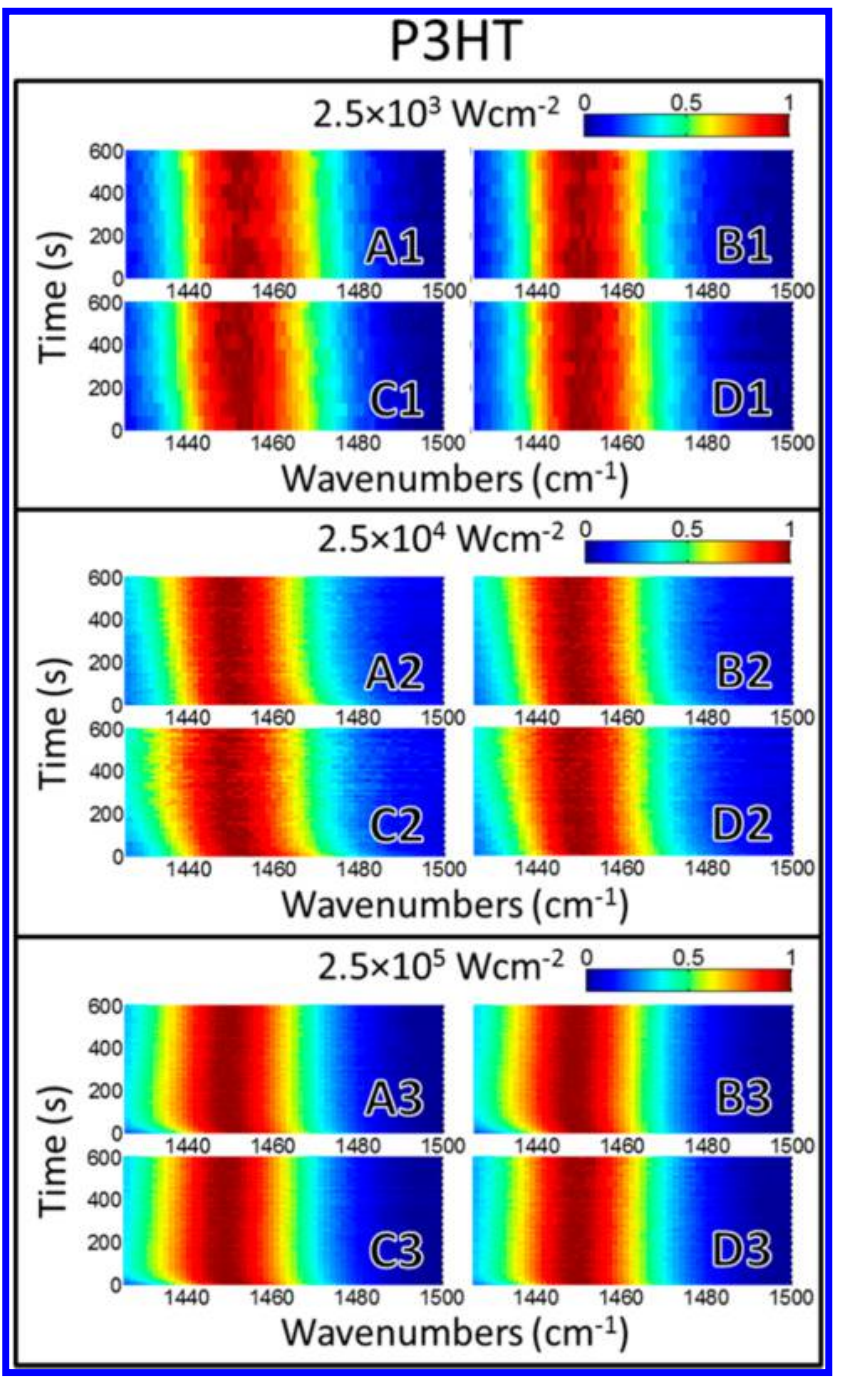

Figure 4. An expanded view of normalized P3HT Raman spectra versus illumination time for three laser power densities listed above each set of graphs. Thick film with solvent evaporation under nitrogen (A1-A3), thick film with slow solvent evaporation under nitrogen (B1-B3), thin film with solvent evaporation under nitrogen (C1C3), and a thin film dried under nitrogen followed by thermal annealing (D1-D3). The color scale is Raman scattering intensity.

of $\mathrm{P} 3 \mathrm{HT}$; the background luminescence dominates the spectrum (data not shown). Background luminescence is decreased and P3HT Raman peaks are measured for P3HT or P3HT: $\mathrm{PC}_{60} \mathrm{BM}$ solid thin films. The intensity of background luminescence is thus dependent on many properties including the bulk heterojunction structure. Changes in peak widths or locations are best observed in the spectra that have been background subtracted and normalized to the most intense peak in the spectra (Figure 3, right column).

For $\mathrm{P} 3 \mathrm{HT}$, a 33\% decrease in the Raman peak intensity and a $38 \%$ decrease in luminescence over $112 \mathrm{~s}$ of illumination with $2.5 \times 10^{5} \mathrm{Wcm}^{-2}$ is followed by a relatively stable Raman signal and slightly increased background over the remaining illumination time (Figure 3, D1). The decrease in the Raman signal with continuous illumination indicates that less P3HT is measured over the analysis time. This could be the result of P3HT photodegradation (i.e., alterations in the chemical structure) or changes to the thickness of the film. Atomic force microscopy (AFM) images of the area illuminated by 2.5 


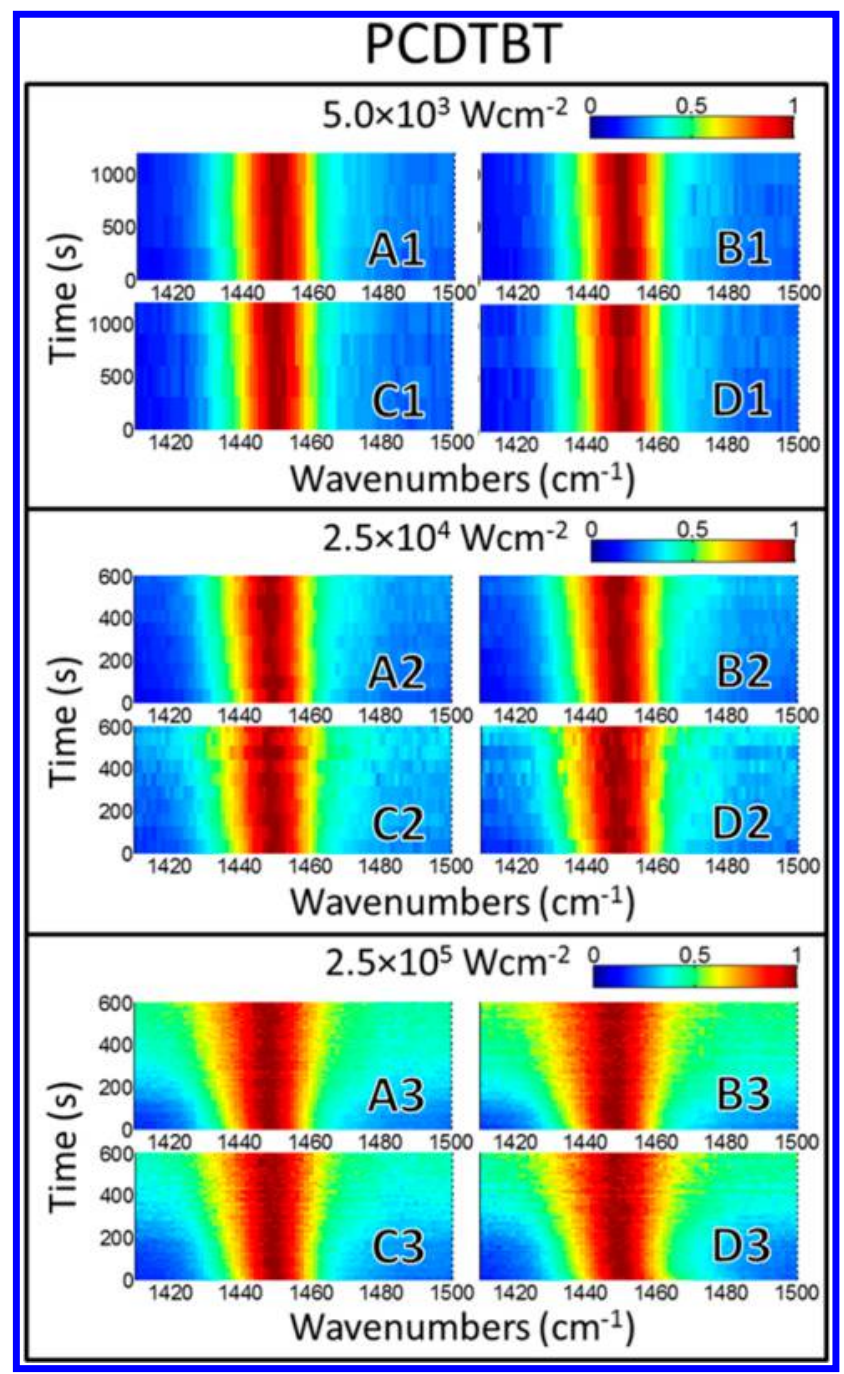

Figure 5. An expanded view of normalized PCDTBT Raman spectra versus illumination time for three laser power densities listed above each set of graphs. Thick film with solvent evaporation under nitrogen $(\mathrm{A} 1-\mathrm{A} 3)$, thick film with slow solvent evaporation under nitrogen (B1-B3), thin film with solvent evaporation under nitrogen (C1$\mathrm{C} 3$ ), and a thin film dried under nitrogen followed by thermal annealing (D1-D3). The color scale is Raman scattering intensity.

$\times 10^{5} \mathrm{Wcm}^{-2}$ indicate there are morphological changes to the film as a result of the laser illumination (Figure S1). Approximately $9 \%$ of the polymer is displaced from around the location of illumination to surrounding areas; surprisingly, the area affected is considerably greater than the $1.6 \mu \mathrm{m}$ diameter spot of the laser profile. This suggests that sample heating occurs, affecting an approximately $15 \mu \mathrm{m}$ diameter spot centered around the illuminated area. The amount of polymer displaced by the laser does not entirely explain the $33 \%$ decrease in the Raman signal. Some P3HT photodegradation may be contributing to the initial decrease in Raman signal and background. With $2.5 \times 10^{5} \mathrm{~W} \mathrm{~cm}^{-2}$ illumination the peak fwhm increases from $34.3 \pm 0.1$ to $36.3 \pm 0.4 \mathrm{~cm}^{-1}$ over $600 \mathrm{~s}$; the peak location does not change (Figure 3 D2). The polymer that is not displaced by the laser exhibits decreased order as measured by resonance Raman spectroscopy with $2.5 \times 10^{5} \mathrm{~W}$ $\mathrm{cm}^{-2}$ illumination. The increase in luminescence with an onset time of $75 \mathrm{~s}$ suggests that the bulk heterojunction, or mixing of

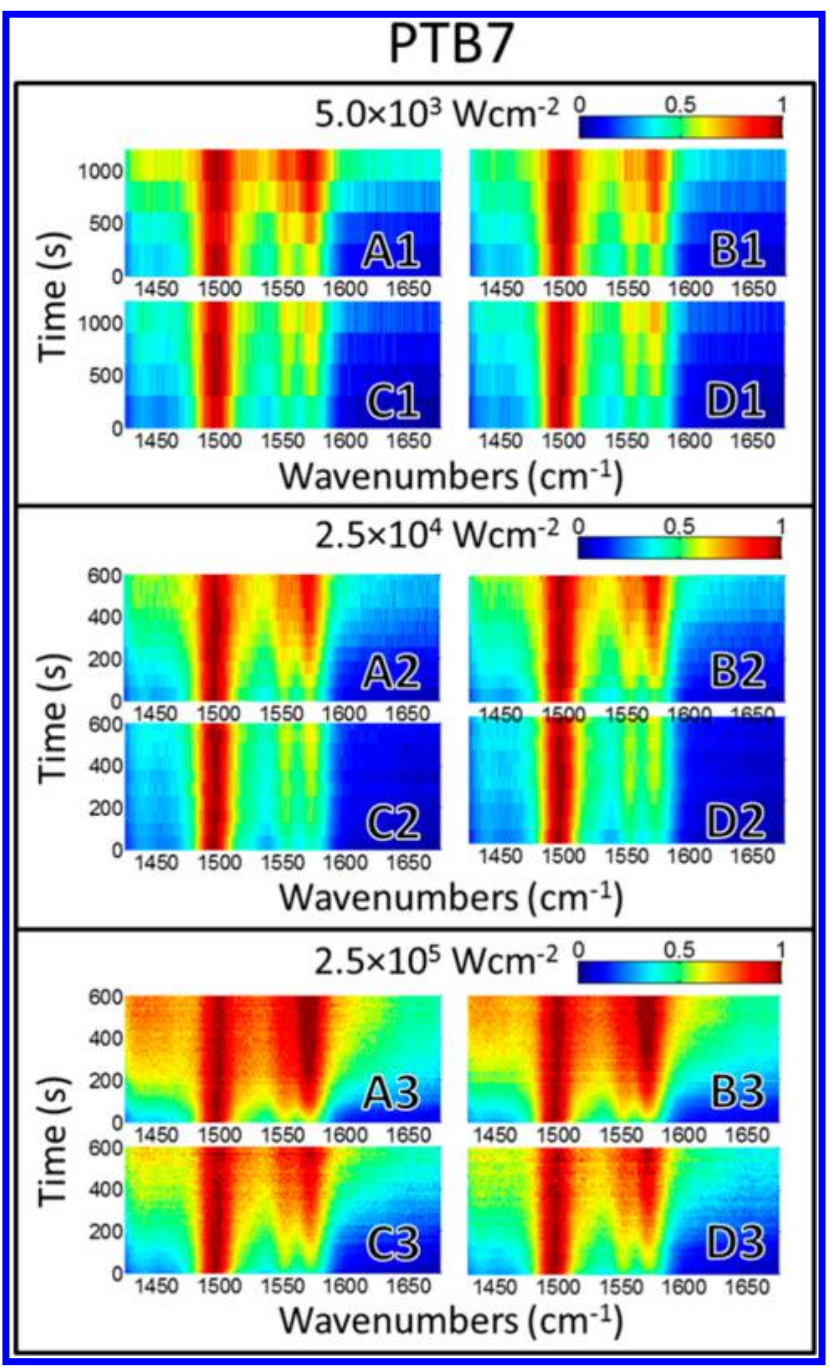

Figure 6. An expanded view of normalized PTB7 Raman spectra versus illumination time for three laser power densities listed above each set of graphs. Thick film with solvent evaporation under nitrogen $(\mathrm{A} 1-\mathrm{A} 3)$, thick film with slow solvent evaporation under nitrogen (B1-B3), thin film with solvent evaporation under nitrogen (C1$\mathrm{C} 3$ ), and a thin film dried under nitrogen followed by thermal annealing (D1-D3). The color scale is Raman scattering intensity.

P3HT and $\mathrm{PC}_{60} \mathrm{BM}$, may be affected by the illumination or resulting sample heating.

The Raman spectra of P3HT show a $14 \%$ decrease in the Raman peak intensity and $22 \%$ decrease in luminescence over $600 \mathrm{~s}$ with $5.0 \times 10^{3} \mathrm{~W} \mathrm{~cm}^{-2}$ illumination (Figure $3 \mathrm{~A} 1$ ). Considering the normalized P3HT spectra, there is no significant change in the $\sim 1450 \mathrm{~cm}^{-1}$ peak location and a slight increase in fwhm from $34.1 \pm 0.7$ to $35.3 \pm 0.4 \mathrm{~cm}^{-1}$ with illumination (Figure 3, A2). No sign of laser-induced changes to the film were visible in optical images after illumination with $5.0 \times 10^{3} \mathrm{~W} \mathrm{~cm}^{-2}$. Some amount of polymer displacement and/or photodegradation explains the decrease in Raman signal and luminescence; however, there are minimal changes to the bulk heterojunction structure when studied with the lower power density. This is consistent with work by Manceau et al. that shows high stability with 1 sun $\left(1000 \mathrm{~W} \mathrm{~m}^{-2}\right)$ over long exposure times for thiophene-based donors, and is also consistent with the work by Tromholt et al. that shows low degradation rates for thick P3HT:PCBM films. ${ }^{34,35}$ 


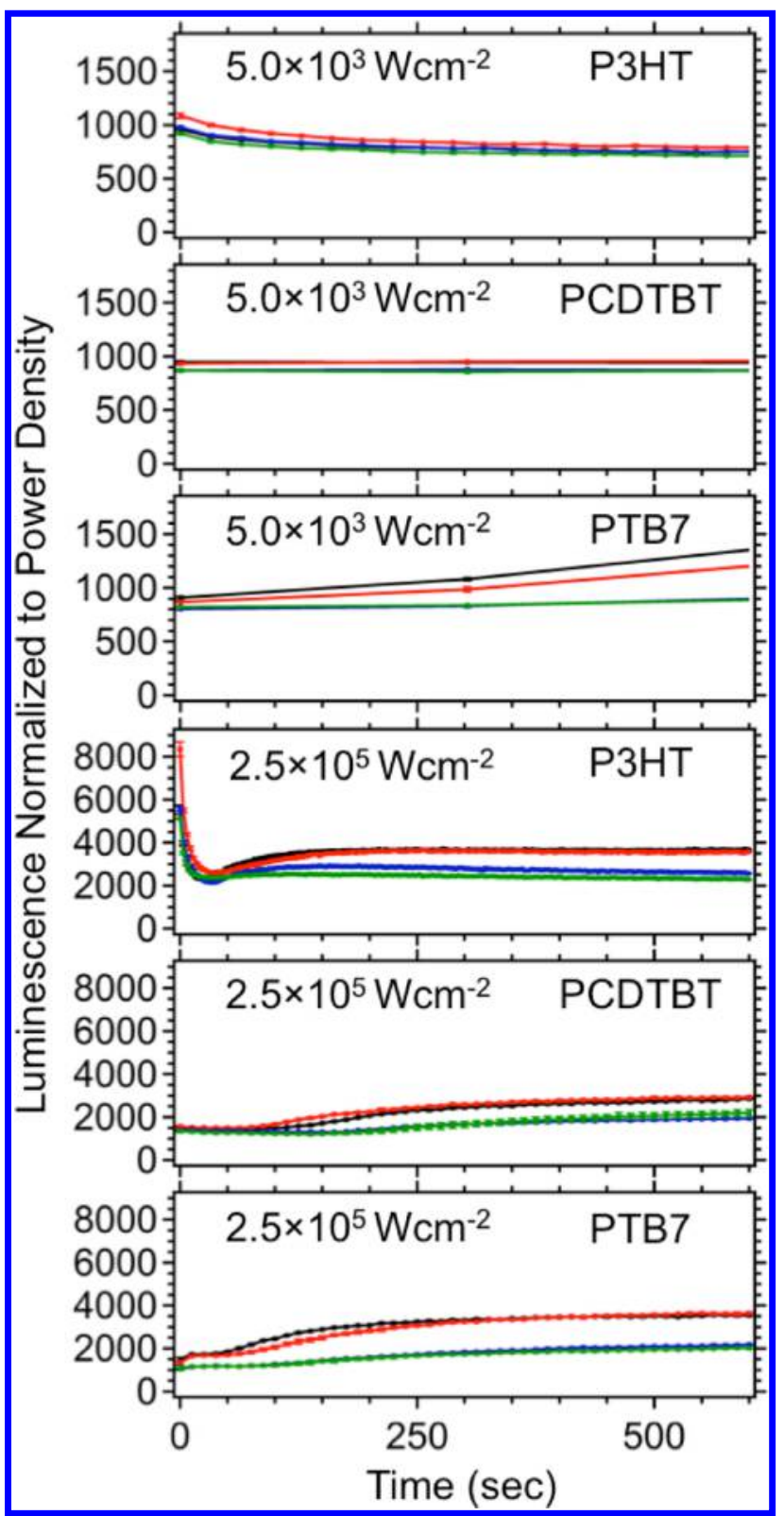

Figure 7. Plots of background luminescence measured at $2000 \mathrm{~cm}^{-1}$ in the Raman spectra versus illumination time for samples fabricated with the indicated donor. The top three graphs were collected using $5.0 \times$ $10^{3} \mathrm{~W} \mathrm{~cm}^{-2}$ and the bottom three graphs with $2.5 \times 10^{5} \mathrm{~W} \mathrm{~cm}^{-2}$. The color code for the samples are thick film with solvent evaporation under nitrogen (black); thick film with slow solvent evaporation under nitrogen (red); thin film with solvent evaporation under nitrogen (blue); and a thin film dried under nitrogen followed by thermal annealing (green). Comparing the results for samples with different preparation, the primary differences in the luminescence intensity are explained by film thickness (Table 1), with the thicker films producing more luminescence than the thinner films in most cases.

The stability of the PCDTBT and PTB7 samples with illumination is fundamentally different compared to the P3HT sample. PCDTBT has a relatively stable Raman intensity, background, peak fwhm, and peak location with $5.0 \times 10^{3} \mathrm{~W}$ $\mathrm{cm}^{-2}$ illumination (Figure 3, B1-B2). With $2.5 \times 10^{5} \mathrm{Wcm}^{-2}$ illumination, the Raman signal is fairly constant, but there is an evolution of luminescence starting after approximately $200 \mathrm{~s}$ of illumination and a $2 \mathrm{~cm}^{-1}$ increased peak fwhm after $50 \mathrm{~s}$ (Figure 3, E1-E2). The AFM image of the illuminated area shows extensive morphology changes to the film with a decrease in polymer thickness localized to the area of illumination (Figure S1). Interestingly, the $82 \%$ decrease in the AFM height images at the area of illumination does not result in a decrease in the Raman intensity. One possibility for the stable PCDTBT Raman intensity could be due to the localized heating causing the $\mathrm{PC}_{70} \mathrm{BM}$ to phase segregate and migrate, which is known to occur during thermal annealing. ${ }^{36}$ The Raman spectra of PTB7 follow similar trends at all power densities as measured for PCDTBT with a $5.0 \times 10^{3} \mathrm{~W} \mathrm{~cm}^{-2}$ illumination (Figure 3, $\mathrm{C} 1-\mathrm{C} 2$ and $\mathrm{F} 1-\mathrm{F} 2$ ). Also, the AFM images of PTB7 after $5.0 \times 10^{3} \mathrm{~W} \mathrm{~cm}^{-2}$ illumination are nearly identical to the P3HT AFM image, both showing an approximately $9 \%$ displacement of the polymer film extending beyond the illuminated area. For both PCDTBT and PTB7, the relatively stable Raman intensity, increased fwhm, and increase in luminescence suggest a less favorable bulk heterojunction structure results with illumination or associated sample heating.

When performing laser-based measurements of thin films, the power density should be low enough to avoid influencing the measurement unless photostability is the property of interest. Laser-based measurements can provide false information as a result of the laser illumination used to generate the data. The laser power and illumination area (i.e., power density) should be considered as well as the duration of illumination. High NA objectives are especially problematic as they focus the light to a small spot and can lead to substantial laser power densities. Power densities of $2.5 \times 10^{3}$ to $2.5 \times 10^{5}$ $\mathrm{Wcm}^{-2}$ with a $1.6 \pm 0.2 \mu \mathrm{m}$ diameter excitation spot size were used in this work (only data for selected, representative power densities have been included in the figures and tables). As discussed above, changes to the films were measured within seconds for P3HT, PCDTBT, and PTB7 at the higher end of the power density range used in this study, which is similar to the $\sim 10^{5} \mathrm{~W} \mathrm{~cm} \mathrm{~cm}^{-2}$ power densities with visible excitation wavelengths that have been used in some Raman studies of polymer order. ${ }^{22,23}$ Recent work indicates that the film thickness can affect degradation rates for a polymer donor. $^{35,37,38}$ Two thickness ranges were included for each polymer donor in the present study; photostability rates may vary for other film thicknesses.

Bulk Heterojunction Thin Film Order and Stability as a Function of Sample Preparation Method. Raman measurements were performed to determine the effect of preparation conditions on the polymer order in bulk heterojunction thin films. The peak fwhm was measured with $2.5 \times 10^{3}(\mathrm{P} 3 \mathrm{HT})$ or $5.0 \times 10^{3}\left(\right.$ PCDTBT and PTB7) $\mathrm{W} \mathrm{cm} \mathrm{cm}^{-2}$ and a $3.5 \mathrm{~s}$ acquisition and illumination time (Table 1). In these studies, peak fwhm exhibited greater changes than peak location from one sample to the next. P3HT shows significant changes to polymer order with varying sample preparation methods; this is consistent with other analysis methods. ${ }^{21,39-41}$ The relative order is highest for the film that was slowly dried by solvent annealing under nitrogen, and decreases for the film that was thermally annealed after drying under nitrogen. The least ordered are the thin and thick films dried under nitrogen without further processing. Supporting Information Figure S2 corresponds to $32 \times 32 \mu \mathrm{m}$ Raman images generated from fwhm of P3HT spectra measured with a power density of $2.5 \times$ $10^{3} \mathrm{~W} \mathrm{~cm}^{-2}$. Each image corresponds to one of the sample preparation methods studied. While the order varies for samples with different preparation conditions, as already discussed, the $0.3 \mathrm{~cm}^{-1}$ standard deviation in the fwhm 
Table 2. Raman Peak Parameters Measured at the Start of the Illumination and after 600 s of Illumination for Samples with the Indicated Donor, Preparation Condition, and Power Density

\begin{tabular}{|c|c|c|c|c|c|c|}
\hline polymer & sample & $\begin{array}{l}\text { power density (W } \\
\mathrm{cm}^{-2} \text { ) }\end{array}$ & $\begin{array}{c}\text { fwhm }\left(\mathrm{cm}^{-1}\right) \text { at } \\
t_{0}\end{array}$ & $\begin{array}{c}\text { fwhm }\left(\mathrm{cm}^{-1}\right) \text { at } \\
t_{\text {end }}\end{array}$ & 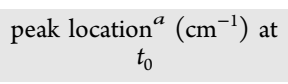 & $\begin{array}{c}\text { peak location }\left(\mathrm{cm}^{-1}\right) \text { at } \\
t_{\text {end }}\end{array}$ \\
\hline \multirow[t]{12}{*}{ P3HT } & \multirow[t]{3}{*}{ fast dry thick } & $2.5 \times 10^{3}$ & $34.1 \pm 0.7$ & $35.3 \pm 0.4$ & 1454 & 1452 \\
\hline & & $2.5 \times 10^{4}$ & $34.4 \pm 0.2$ & $35.1 \pm 0.3$ & 1453 & 1449 \\
\hline & & $2.5 \times 10^{5}$ & $34.3 \pm 0.1$ & $36.3 \pm 0.4$ & 1452 & 1449 \\
\hline & \multirow[t]{3}{*}{ slow dry thick } & $2.5 \times 10^{3}$ & $31.3 \pm 0.4$ & $31.23 \pm 0.09$ & 1451 & 1451 \\
\hline & & $2.5 \times 10^{4}$ & $31.3 \pm 0.2$ & $34.8 \pm 0.8$ & 1451 & 1447 \\
\hline & & $2.5 \times 10^{5}$ & $32.03 \pm 0.09$ & $36.4 \pm 0.5$ & 1450 & 1448 \\
\hline & \multirow[t]{3}{*}{ fast dry thin } & $2.5 \times 10^{3}$ & $34.73 \pm 0.07$ & $34.0 \pm 0.9$ & 1454 & 1451 \\
\hline & & $2.5 \times 10^{4}$ & $35.4 \pm 0.3$ & $37.8 \pm 0.8$ & 1453 & 1448 \\
\hline & & $2.5 \times 10^{5}$ & $34.1 \pm 0.3$ & $36.4 \pm 0.05$ & 1451 & 1448 \\
\hline & \multirow[t]{3}{*}{ fast dry thermal anneal thin } & $2.5 \times 10^{3}$ & $32.2 \pm 0.3$ & $32.2 \pm 0.5$ & 1452 & 1451 \\
\hline & & $2.5 \times 10^{4}$ & $32.6 \pm 0.1$ & $36.8 \pm 0.5$ & 1451 & 1447 \\
\hline & & $2.5 \times 10^{5}$ & $33.6 \pm 0.2$ & $36 \pm 1$ & 1450 & 1449 \\
\hline \multirow[t]{12}{*}{ PCDTBT } & \multirow[t]{3}{*}{ fast dry thick } & $5.0 \times 10^{3}$ & $22.2 \pm 0.6$ & $22.9 \pm 0.6$ & 1449 & 1448 \\
\hline & & $2.5 \times 10^{4}$ & $22.1 \pm 0.2$ & $24.0 \pm 0.3$ & 1449 & 1447 \\
\hline & & $2.5 \times 10^{5}$ & $23.0 \pm 0.1$ & $25 \pm 1$ & 1448 & 1446 \\
\hline & \multirow[t]{3}{*}{ slow dry thick } & $5.0 \times 10^{3}$ & $22.2 \pm 0.3$ & $23.3 \pm 0.4$ & 1449 & 1448 \\
\hline & & $2.5 \times 10^{4}$ & $22.2 \pm 0.2$ & $23.8 \pm 0.2$ & 1449 & 1447 \\
\hline & & $2.5 \times 10^{5}$ & $23.7 \pm 0.2$ & $25 \pm 1$ & 1447 & 1446 \\
\hline & \multirow[t]{3}{*}{ fast dry thin } & $5.0 \times 10^{3}$ & $22.0 \pm 0.6$ & $21.8 \pm 0.8$ & 1449 & 1448 \\
\hline & & $2.5 \times 10^{4}$ & $23.0 \pm 0.7$ & $26 \pm 1$ & 1448 & 1447 \\
\hline & & $2.5 \times 10^{5}$ & $22.4 \pm 0.1$ & $27.3 \pm 0.9$ & 1449 & 1447 \\
\hline & \multirow[t]{3}{*}{ fast dry thermal anneal thin } & $5.0 \times 10^{3}$ & $22.5 \pm 0.8$ & $22.9 \pm 0.6$ & 1449 & 1448 \\
\hline & & $2.5 \times 10^{4}$ & $22.9 \pm 0.2$ & $24.9 \pm 0.2$ & 1448 & 1447 \\
\hline & & $2.5 \times 10^{5}$ & $26 \pm 3$ & $27 \pm 2$ & 1451 & 1447 \\
\hline
\end{tabular}

indicates a high degree of uniformity across a sample. This is expected since the typical P3HT or PCBM domain size measured for samples with similar preparations conditions is reported to be considerably smaller (e.g., nanoscale) than the diameter of the laser spot used to collect the Raman images. ${ }^{5}$ Absorption spectra are susceptible to changes in isolated, ordered and disordered phases of P3HT. ${ }^{42}$ Peaks at 550 and $600 \mathrm{~nm}$ are assigned to ordered phases, while peaks at higher energies correspond to isolated and disordered phases. The Raman fwhm values (Table 1) and absorption spectra (Figure 2 ), when normalized to account for differences in film thickness, show relative agreement on the degree of polymer order for the $\mathrm{P} 3 \mathrm{HT}$ films prepared with different preparation conditions.

In the case of PCDTBT and PTB7 there are no statistically significant differences in the fwhm measured for samples with different preparation conditions (Table 1). Raman spectroscopy measurements show that the PCDTBT film is not affected by thermal annealing at $80^{\circ}$. This finding is supported by neutron reflectivity and grazing incidence wide-angle $\mathrm{X}$-ray scattering measurements that showed thermal annealing at $70^{\circ}$ has a negligible effect on PCDTBT order. ${ }^{43}$ Consistent with the Raman data, PCDTBT and PTB7 show minimal changes in their absorption spectra for samples with different preparation conditions when sample thickness is taken into account (Figure 2).

Photostability was tested for each polymer donor and sample preparation method. The Raman spectra collected for the four sample preparation methods and three power densities are shown in Figure 4 for P3HT, Figure 5 for PCDTBT, and Figure 6 for PTB7. Qualitatively, the Raman spectral changes and luminescence (Figure 7) are similar to the trends that have already been discussed and are independent of sample preparation method for a given polymer donor.

A quantitative measure of peak properties was performed for P3HT and PCDTBT (Table 2). The background in the PTB7 spectra produced large uncertainties at long illumination times, particularly for the highest power density, and further quantification of peak properties was not performed. Despite having different fwhm values at the start of illumination, the fwhm measured for all four sample preparation methods are statistically similar after illumination with the highest power density for P3HT. This indicates that regardless of the effect of preparation conditions on the initial order, the resulting order after illumination is not dependent on the preparation conditions. This is not true for PCDTBT, which shows a correlation between film thickness and polymer order after illumination at the higher power densities. The two thinnest samples had the greatest relative disorder (fwhm $27.3 \pm 0.9$ and $27 \pm 2 \mathrm{~cm}^{-1}$ ) measured after illumination for power densities greater than $2.5 \times 10^{4} \mathrm{~W} \mathrm{~cm}^{-2}$.

\section{CONCLUSIONS}

Laser-based studies of P3HT, PCDTBT, and PTB7 using high laser power densities can degrade the morphology or bulk heterojunction structure, which will invalidate the results of the study. This work highlights power densities and exposure times that lead to reliable resonance Raman measurements of polymer order, which varies with preparation conditions for P3HT but not PCDTBT or PTB7. Also, the measured photostability varied for P3HT, PCDTBT, and PTB7. The photostability after prolonged illumination with the highest power density was not significantly different for the sample preparation conditions studied for P3HT; however, PCDTBT 
may exhibit a thickness-dependent stability with illumination. This information can be used to design more stable devices, with the end goal of OPVs suitable for a range of real-world applications.

\section{ASSOCIATED CONTENT}

\section{S Supporting Information}

Optical and AFM images (Figure S1) of a $2.5 \times 10^{5} \mathrm{~W} \mathrm{~cm}^{-2}$ illuminated area on P3HT, PCDTBT, and PTB7 films. Figure $\mathrm{S} 2$ is the Raman images of $\mathrm{P} 3 \mathrm{HT}: \mathrm{PC}_{60} \mathrm{BM}$ films. This material is available free of charge via the Internet at http://pubs.acs.org.

\section{AUTHOR INFORMATION}

\section{Corresponding Author}

*E-mail: esmith1@iastate.edu.

\section{Author Contributions}

\#Both authors contributed equally.

Notes

The authors declare no competing financial interest.

\section{ACKNOWLEDGMENTS}

Raman measurements and film characterization was supported by the U.S. Department of Energy, Office of Basic Energy Sciences, Division of Chemical Sciences, Geosciences, and Biosciences through the Ames Laboratory. The Ames Laboratory is operated for the U.S. Department of Energy by Iowa State University under Contract No. DE-AC0207CH11358. Film preparation was supported by NSF ECCS1055930. M.E. was supported by the Egyptian Government through Grant GM915.

\section{REFERENCES}

(1) Lizin, S.; Van Passel, S.; De Schepper, E.; Vranken, L. The Future of Organic Photovoltaic Solar Cells as a Direct Power Source for Consumer Electronics. Sol. Energy Mater. Sol. Cells 2012, 103, 1-10.

(2) García-Valverde, R.; Cherni, J. A.; Urbina, A. Life Cycle Analysis of Organic Photovoltaic Technologies. Prog. Photovoltaics: Res. Appl. 2010, 18, 535-558.

(3) Carlé, J. E.; Krebs, F. C. Technological Status of Organic Photovoltaics (OPV). Sol. Energy Mater. Sol. Cells 2013, 119, 309-310.

(4) Aïch, B. R.; Beaupré, S.; Leclerc, M.; Tao, Y. Highly Efficient Thieno[3,4-c]pyrrole-4,6-dione-Based Solar Cells Processed from Non-chlorinated Solvent. Org. Electron. 2014, 15, 543-548.

(5) Chen, D.; Nakahara, A.; Wei, D.; Nordlund, D.; Russell, T. P. P3HT/PCBM Bulk Heterojunction Organic Photovoltaics: Correlating Efficiency and Morphology. Nano Lett. 2010, 11, 561-567.

(6) Grossiord, N.; Kroon, J. M.; Andriessen, R.; Blom, P. W. Degradation Mechanisms in Organic Photovoltaic Devices. Org. Electron. 2012, 13, 432-456.

(7) Gregg, B. A. Charged Defects in Soft Semiconductors and Their Influence on Organic Photovoltaics. Soft Matter 2009, 5, 2985-2989.

(8) Wang, H.; Fukuda, T.; Ishikawa, N.; Matsuo, Y. SolventDependent Morphology of Thermally Converted Copper Phthalocyanine for Solution-Processed Small Molecule Organic Photovoltaic Devices. Org. Electron. 2014, 15, 139-143.

(9) Brown, P. J.; Thomas, D. S.; Köhler, A.; Wilson, J. S.; Kim, J.-S.; Ramsdale, C. M.; Sirringhaus, H.; Friend, R. H. Effect of Interchain Interactions on the Absorption and Emission of Poly(3-Hexylthiophene). Phys. Rev. B 2003, 67, 064203.

(10) Zhang, Y.; Yip, H.-L.; Acton, O.; Hau, S. K.; Huang, F.; Jen, A. K. Y. A Simple and Effective Way of Achieving Highly Efficient and Thermally Stable Bulk-Heterojunction Polymer Solar Cells Using Amorphous Fullerene Derivatives as Electron Acceptor. Chem. Mater. 2009, 21, 2598-2600.
(11) Zimmermann, B.; Würfel, U.; Niggemann, M. Longterm Stability of Efficient Inverted P3HT:PCBM Solar Cells. Sol. Energy Mater. Sol. Cells 2009, 93, 491-496.

(12) Hsieh, C.-H.; Cheng, Y.-J.; Li, P.-J.; Chen, C.-H.; Dubosc, M.; Liang, R.-M.; Hsu, C.-S. Highly Efficient and Stable Inverted Polymer Solar Cells Integrated with a Cross-Linked Fullerene Material as an Interlayer. J. Am. Chem. Soc. 2010, 132, 4887-4893.

(13) He, D.; Du, X.; Zhang, W.; Xiao, Z.; Ding, L. Improving the Stability of $\mathrm{P} 3 \mathrm{HT} / \mathrm{PC}_{61} \mathrm{BM}$ Solar Cells by a Thermal Crosslinker. J. Mater. Chem. A 2013, 1, 4589-4594.

(14) Mateker, W. R.; Douglas, J. D.; Cabanetos, C.; Sachs-Quintana, I. T.; Bartelt, J. A.; Hoke, E. T.; El Labban, A.; Beaujuge, P. M.; Frechet, J. M. J.; McGehee, M. D. Improving the Long-Term Stability of Pbdttpd Polymer Solar Cells through Material Purification Aimed at Removing Organic Impurities. Energy Environ. Sci. 2013, 6, 25292537.

(15) Xu, X.-J.; Yang, L.-Y.; Tian, H.; Qin, W.-J.; Yin, S.-G.; Zhang, F. Enhanced Performance and Stability in Polymer Photovoltaic Cells Using Ultraviolet-Treated PEDOT:PSS. Chin. Phys. Lett. 2013, 30, 077201.

(16) Gevorgyan, S. A.; Jørgensen, M.; Krebs, F. C. A Setup for Studying Stability and Degradation of Polymer Solar Cells. Sol. Energy Mater. Sol. Cells 2008, 92, 736-745.

(17) Krebs, F. C. Air Stable Polymer Photovoltaics Based on a Process Free from Vacuum Steps and Fullerenes. Sol. Energy Mater. Sol. Cells 2008, 92, 715-726.

(18) Krebs, F. C.; Spanggaard, H. Significant Improvement of Polymer Solar Cell Stability. Chem. Mater. 2005, 17, 5235-5237.

(19) An, L.; Duan, Y.; Yuan, Y.; Zhou, L.; Zhang, J. Effect of Thermal Annealing on the Microstructure of P3HT Thin Film Investigated by RAIR Spectroscopy. Vib. Spectrosc. 2013, 68, 40-44.

(20) Carach, C.; Gordon, M. J. Optical Measures of Thermally Induced Chain Ordering and Oxidative Damage in Polythiophene Films. J. Phys. Chem. B 2013, 117, 1950-1957.

(21) Miller, S.; Fanchini, G.; Lin, Y.-Y.; Li, C.; Chen, C.-W.; Su, W.F.; Chhowalla, M. Investigation of Nanoscale Morphological Changes in Organic Photovoltaics During Solvent Vapor Annealing. J. Mater. Chem. 2008, 18, 306-312.

(22) Gao, Y.; Grey, J. K. Resonance Chemical Imaging of Polythiophene/Fullerene Photovoltaic Thin Films: Mapping Morphology-Dependent Aggregated and Unaggregated $\mathrm{C}=\mathrm{C}$ Species. J. Am. Chem. Soc. 2009, 131, 9654-9662.

(23) Gao, Y.; Martin, T. P.; Thomas, A. K.; Grey, J. K. Resonance Raman Spectroscopic- and Photocurrent Imaging of Polythiophene/ Fullerene Solar Cells. J. Phys. Chem. Lett. 2009, 1, 178-182.

(24) Tsoi, W. C.; James, D. T.; Kim, J. S.; Nicholson, P. G.; Murphy, C. E.; Bradley, D. D.; Nelson, J.; Kim, J. S. The Nature of In-Plane Skeleton Raman Modes of P3HT and Their Correlation to the Degree of Molecular Order in P3HT:PCBM Blend Thin Films. J. Am. Chem. Soc. 2011, 133, 9834-43.

(25) Veerender, P.; Saxena, V.; Chauhan, A. K.; Koiry, S. P.; Jha, P.; Gusain, A.; Choudhury, S.; Aswal, D. K.; Gupta, S. K. Probing the Annealing Induced Molecular Ordering in Bulk Heterojunction Polymer Solar Cells Using in-Situ Raman Spectroscopy. Sol. Energy Mater. Sol. Cells 2014, 120, 526-535.

(26) Meyer, M. W.; Larson, K. L.; Mahadevapuram, R. C.; Lesoine, M. D.; Carr, J. A.; Chaudhary, S.; Smith, E. A. Scanning Angle Raman Spectroscopy of Poly(3-Hexylthiophene)-Based Films on Indium Tin Oxide, Gold, and Sapphire Surfaces. ACS Appl. Mater. Interfaces 2013, 5, 8686-8693.

(27) Yun, J.-J.; Peet, J.; Cho, N.-S.; Bazan, G. C.; Lee, S. J.; Moskovits, M. Insight into the Raman Shifts and Optical Absorption Changes Upon Annealing Polymer/Fullerene Solar Cells. Appl. Phys. Lett. 2008, 92, 251912.

(28) Furukawa, Y.; Seto, K.; Nakajima, K.; Itoh, Y.; Eguchi, J.; Sugiyama, T.; Fujimura, H. Infrared and Raman Spectroscopy of Organic Thin Films Used for Electronic Devices. Vib. Spectrosc 2012, $60,5-9$. 
(29) Furukawa, Y. Vibrational Spectroscopy of Organic Thin Films Used for Solar Cells. AIP Conf. Proc. 2013, 1554, 5-8.

(30) Wood, S.; Garnett, O.; Tokmoldin, N.; Tsoi, W. C.; Haque, S. A.; Kim, J.-S. In Situ Formation of Organic-Inorganic Hybrid Nanostructures for Photovoltaic Applications. Faraday Discuss. 2014, DOI: $10.1039 / C 4 F D 00141 \mathrm{~A}$.

(31) Wang, X.; Zhang, D.; Braun, K.; Egelhaaf, H.-J.; Brabec, C. J.; Meixner, A. J. High-Resolution Spectroscopic Mapping of the Chemical Contrast from Nanometer Domains in P3HT:PCBM Organic Blend Films for Solar-Cell Applications. Adv. Funct. Mater. 2010, 20, 492-499.

(32) Reish, M. E.; Nam, S.; Lee, W.; Woo, H. Y.; Gordon, K. C. A Spectroscopic and DFT Study of the Electronic Properties of Carbazole-Based D-A Type Copolymers. J. Phys. Chem. C 2012, $116,21255-21266$.

(33) Jameh-Bozorghi, S.; Il Beigi, H. S. Theoretical Study on the Electronic, Structural, Properties and Reactivity of a Series of Mono-, Di-, Tri- and Tetrafluorothiophenes as Monomers for New Conducting Polymers. J. Fluorine Chem. 2011, 132, 190-195.

(34) Manceau, M.; Bundgaard, E.; Carle, J. E.; Hagemann, O.; Helgesen, M.; Sondergaard, R.; Jorgensen, M.; Krebs, F. C. Photochemical Stability of $\pi$-Conjugated Polymers for Polymer Solar Cells: A Rule of Thumb. J. Mater. Chem. 2011, 21, 4132-4141.

(35) Tromholt, T.; Madsen, M. V.; Carle, J. E.; Helgesen, M.; Krebs, F. C. Photochemical Stability of Conjugated Polymers, Electron Acceptors and Blends for Polymer Solar Cells Resolved in Terms of Film Thickness and Absorbance. J. Mater. Chem. 2012, 22, 75927601.

(36) Watts, B.; Belcher, W. J.; Thomsen, L.; Ade, H.; Dastoor, P. C. A Quantitative Study of PCBM Diffusion During Annealing of P3HT:PCBM Blend Films. Macromolecules 2009, 42, 8392-8397.

(37) Madsen, M. V.; Tromholt, T.; Norrman, K.; Krebs, F. C. Concentrated Light for Accelerated Photo Degradation of Polymer Materials. Adv. Energy Mater. 2013, 3, 424-427.

(38) Madsen, M. V.; Tromholt, T.; Böttiger, A.; Andreasen, J. W.; Norrman, K.; Krebs, F. C. Influence of Processing and Intrinsic Polymer Parameters on Photochemical Stability of Polythiophene Thin Films. Polym. Degrad. Stab. 2012, 97, 2412-2417.

(39) Campoy-Quiles, M.; Ferenczi, T.; Agostinelli, T.; Etchegoin, P. G.; Kim, Y.; Anthopoulos, T. D.; Stavrinou, P. N.; Bradley, D. D. C.; Nelson, J. Morphology Evolution Via Self-Organization and Lateral and Vertical Diffusion in Polymer:Fullerene Solar Cell Blends. Nat. Mater. 2008, 7, 158-164.

(40) Ebadian, S.; Gholamkhass, B.; Shambayati, S.; Holdcroft, S.; Servati, P. Effects of Annealing and Degradation on Regioregular Polythiophene-Based Bulk Heterojunction Organic Photovoltaic Devices. Sol. Energy Mater. Sol. Cells 2010, 94, 2258-2264.

(41) Lichang, Z.; Tang, C. W.; Chen, S. H. Effects of Active Layer Thickness and Thermal Annealing on Polythiophene: Fullerene Bulk Heterojunction Photovoltaic Devices. Appl. Phys. Lett. 2010, 97, 053305 .

(42) Hu, R.; Zhang, W.; Wang, P.; Qin, Y.; Liang, R.; Fu, L.-M.; Zhang, J.-P.; Ai, X.-C. Characterization and Distribution of Poly(3Hexylthiophene) Phases in an Annealed Blend Film. ChemPhysChem 2014, 15, 935-941.

(43) Staniec, P. A.; et al. The Nanoscale Morphology of a PCDTBT:PCBM Photovoltaic Blend. Adv. Energy Mater. 2011, 1, 499-504. 